\title{
Endoscopic Anatomy of Hypotympanum Surgical Implications
}

\author{
Rajesh Boddepalli Sreerama Murthy Boddepalli \\ ${ }^{1}$ Department of ENT, Suraksha Health Park Hospital, Marripalem, \\ Visakhapatnam, Andhra Pradesh, India
}

Ann Otol Neurotol ISO 2018;1:58-67

\begin{abstract}
Address for correspondence Sreerama Murthy Boddepalli, DLO, MS, Department of ENT, Suraksha Health Park Hospital, Marripalem, Visakhapatnam, 530018, Andhra Pradesh, India (e-mail: murthy.ent@gmail.com).
\end{abstract}

\begin{abstract}
Keywords

- hypotympanum

- hypotympanotomy

- jugular bulb

- glomus jugulare

- trabeculae

- crotch

- band of Seibermann

- temporal bone dissection

Objective To assess the endomeatal endoscopic morphological anatomy of hypotympanum under local anesthesia and further cadaveric dissection of temporal bone to know complete anatomical details of hypotympanum.

Materials and Methods Two hundred six cases of live operations were studied, these included 160 cases of simple dry perforations for myringoplasty and 46 cases of stapedectomy operations. All operations were performed under local anesthesia using a 0 -degree, 4 -mm endoscope. The hypotympanum was visualized. Twenty wet temporal bones were dissected endoscopically and complete hypotympanic details were studied.

Results Out of 206 cases, 94 cases (45\%) were found to be type A; 51 cases (25\%) were type B and 60 cases (30\%) were type $C$ hypotympanum. Wet cadaveric bones were dissected to visualize the transmeatal endoscopic jugular foramen in four levels (levels 1, 2, 3, and 4).
\end{abstract}

\section{Introduction}

Hypotympanum is so far ignored space in otological surgery but it is very important in glomus jugulare tumors and infra cochlear approaches to petrous apex. It is a space below the annulus tympanicus containing air cells and trabecule (-Figs. 1-3). The lateral wall of hypotympanum is formed by tympanic bone; the medial wall is formed by petrous bone. Posteriorly, it is limited by the styloid complex and facial nerve. It is actually a bony groove below the mesotympanum marked by air cells of various sizes and bony septa called the trabeculae. The upper lateral border of hypotympanum is formed by tympanic ring of the tympanic bone. The upper lateral border of hypotympanum is formed by tympanic ring of the tympanic bone. The medial wall is formed by promontory, these two structures are the only consistent ones. Medially and inferiomedially, the floor of hypotympanum is formed by tympanic bone that meets the petrous part of temporal bone. The shape of the hypotympanum is influenced by protrusion of jugular bulb into tympanic cavity. ${ }^{1}$ The other structure lying anteriomedially is the carotid canal; in majority of cases, the anterior wall of the hypotympanum is formed by petrous bone. Actually, the hypotympanum is developed from saccus posticus.

\section{Materials and Methods}

Two hundred six cases of live operations were studied, these included 160 cases of simple dry perforations for myringoplasty and 46 cases of stapedectomy operations. All operations were performed under local anesthesia using a 0-degree, 4-mm endoscope. The hypotympanum was visualized. Twenty wet temporal bones were dissected endoscopically and complete hypotympanic details were studied.

\section{Discussion}

We examined 206 ear cases, using a 0-degree, 0.4-mm endoscope. These included cases with chronic suppurative otitis media (CSOM), with central perforations CSOM, with 


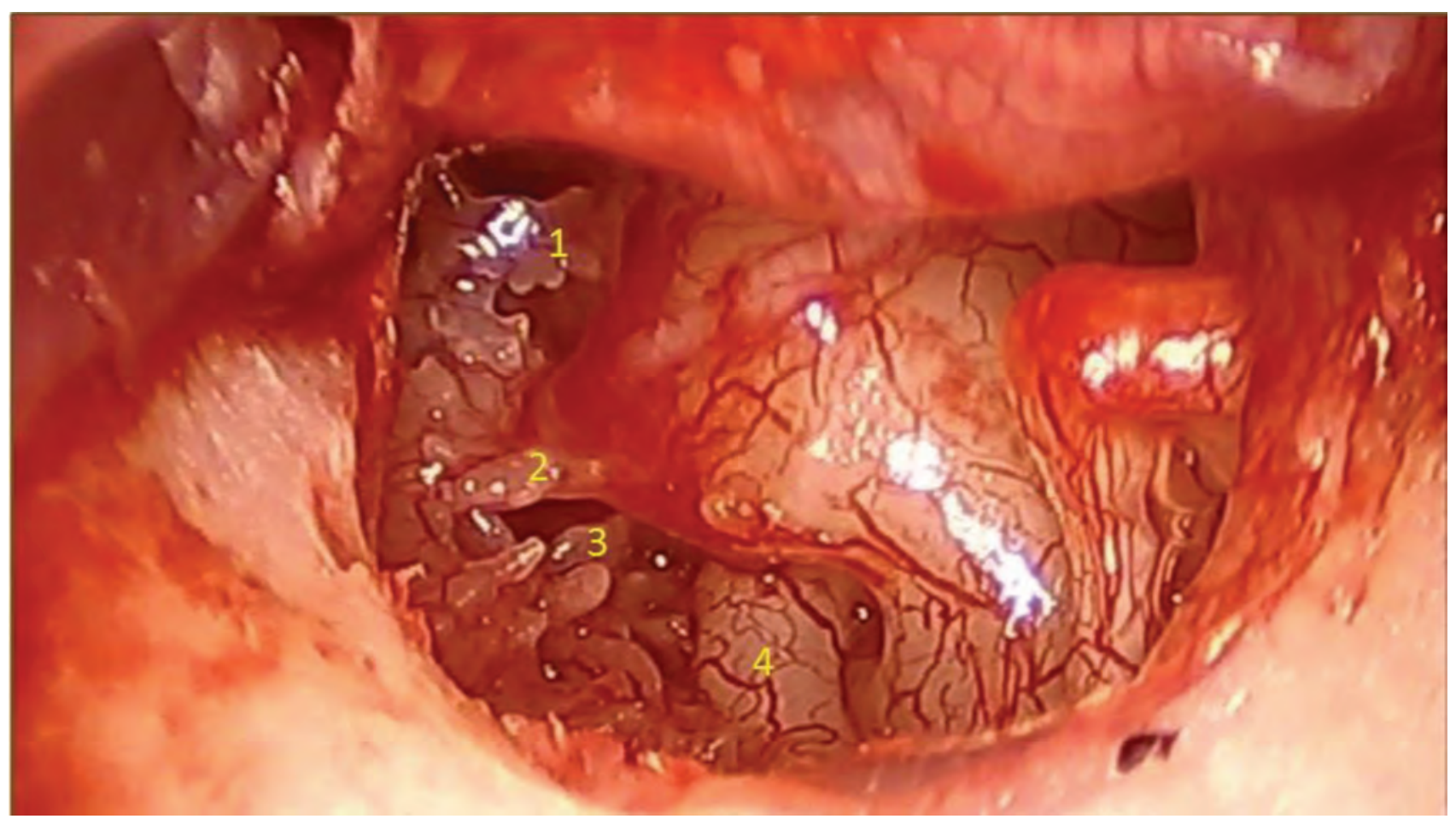

Fig. 1 Anatomy of hypotympanum. 1. Trabeculae longa, 2. Finiculus, 3. Trabeculae profunda, 4. Fustis.

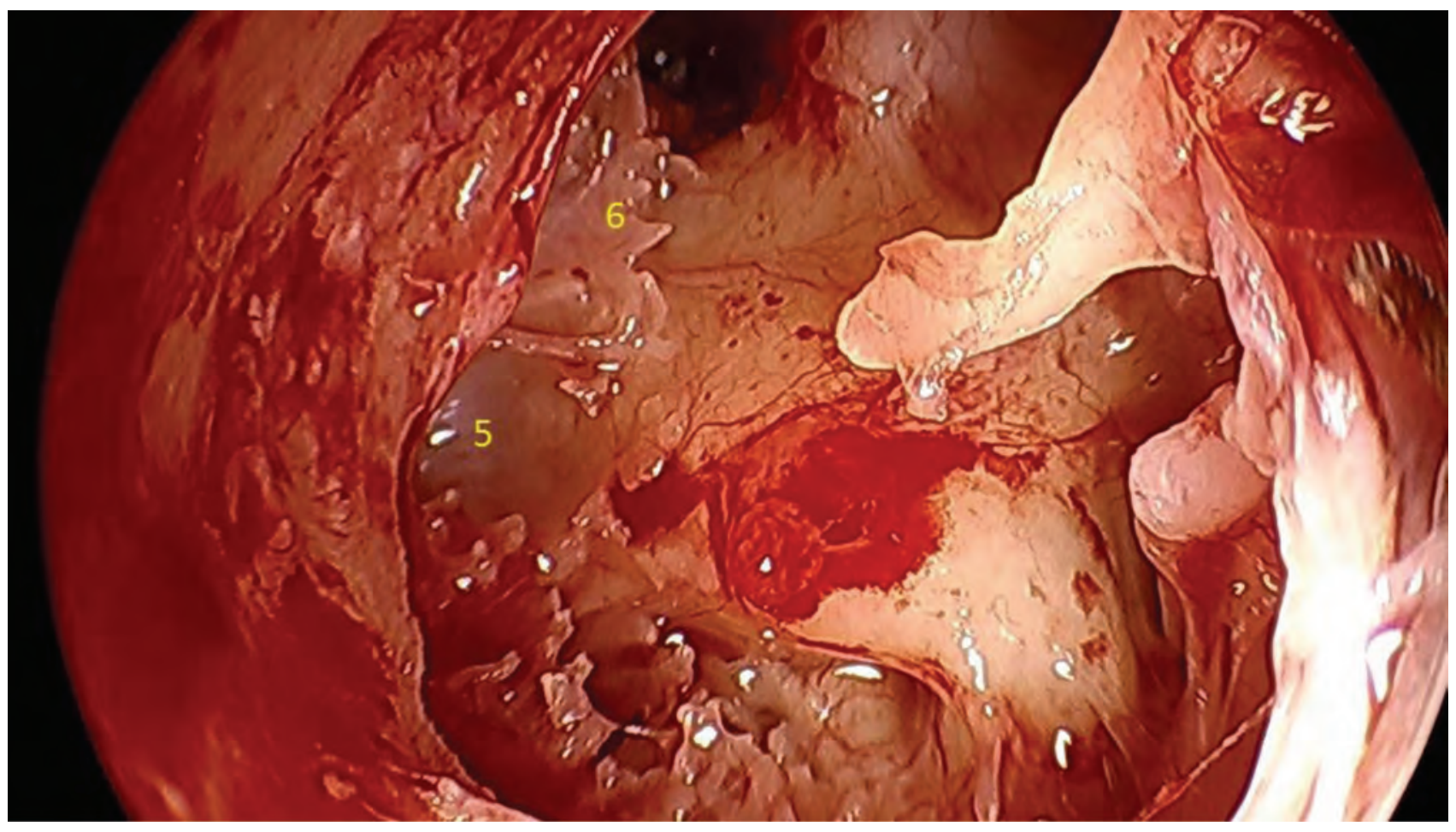

Fig. 2 Anterior hypotympanic sinus. 5. Anterior Hypotympanic Sinus, 6. Protinaculum. 


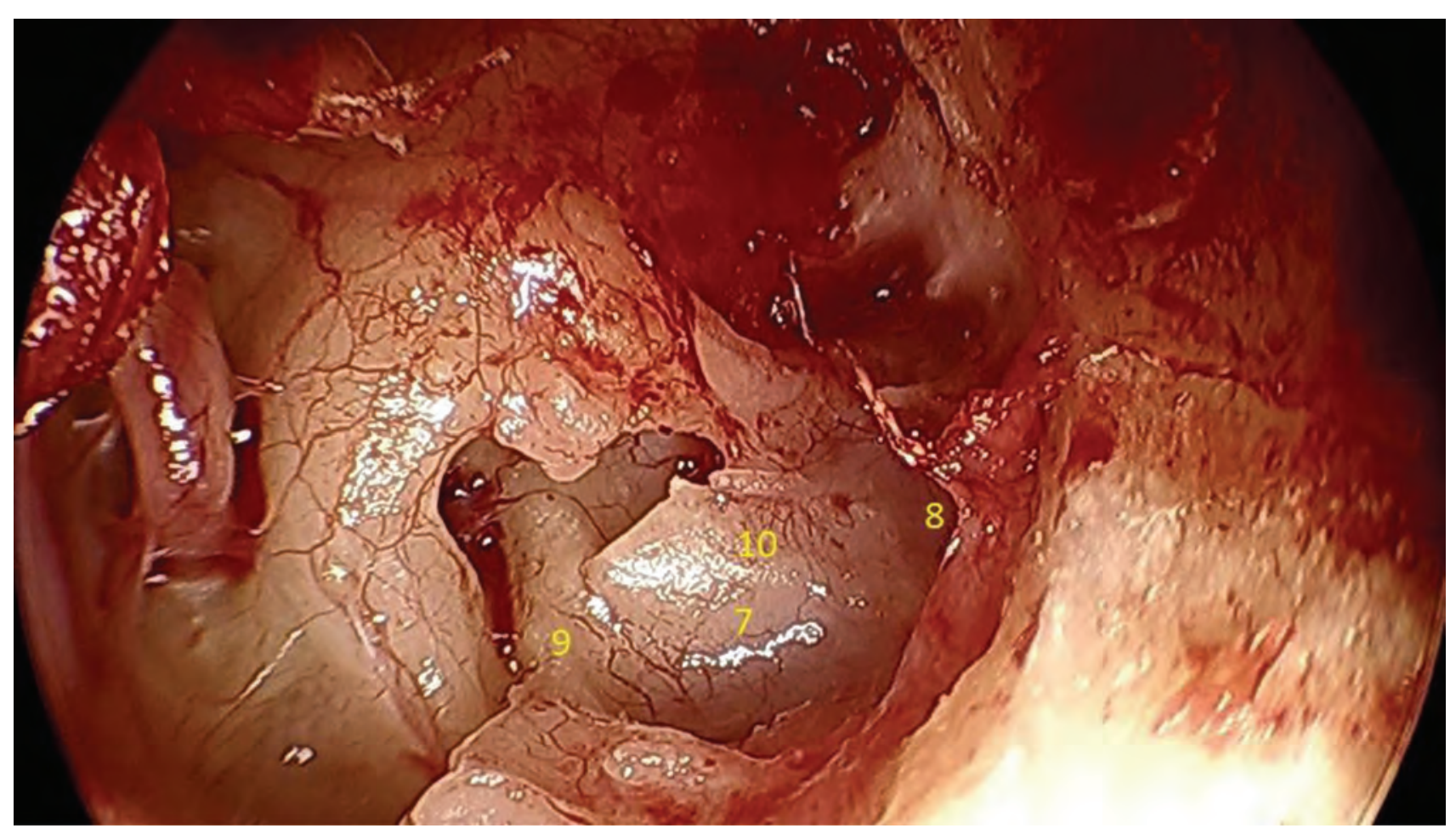

Fig. 3 Posterior hypotympanic sinus. 7. Subfustis sinus, 8. Posterior hypotympanic sinus, 9. Pyramidal leg of fustis, 10. Styloid leg of fustis.

cholesteatoma, and normal ears with otosclerosis. According to our findings, the endoscopic anatomy of hypotympanum is of three types.

\section{Type A Hypotympanum}

Type A hypotympanum is present in $45 \%$ of cases. Normally, the depth of the hypotympanum from the inferior bony annulus to floor of hypotympanum is 8 to $10 \mathrm{~mm}$. It contains air cells and 8 to 10 trabeculae; The anterior most and the longest trabeculae is called the trabeculae longa. It makes the surface marking for crotch jugular carotid septum. We frequently find anterior and the posterior hypotympanum sinuses in these cases. The jugular bulb is very low associated with backward placed sigmoid sinus. ${ }^{2}$ Finiculus is long and associated with infra cochlear space. If this area is well pneumatized, one can almost see the curvature of the basal turn of the cochlea ( - Fig. 4). ${ }^{3}$

\section{Type B Hypotympanum}

Type B hypotympanic sinus is present in 25\% cases; the depth of the hypotympanum is 4 to $5 \mathrm{~mm}$ from the inferior bony tympanic ridge. This type of sinus is associated with sparse trabeculae with limited infracochlear space. Here, the finiculus is short ( $\mathbf{- F i g . 5}$ ).

\section{Type C Hypotympanum}

In type $C$ hypotympanum, the jugular bulb is above the inferior tympanic sulcus. It is present in 30\% of cases; no trabeculae or funiculus is present. In $10 \%$ of these cases, the jugular bulb touches the round window. ${ }^{4}$ There is no infra cochlear space and this type of hypotympanum is associated with forward placed sigmoid sinus (-Fig. 6).

\section{Transmeatal Endoscopic Dissection of Hypotympanum and Jugular Foramen}

In our 206 cases, the majority of hypotympanum were type A hypotympanum. The transmeatal endoscopic dissection in these types of hypotympanum are of four types.

\section{Level 1}

After elevation of tympanomeatal flap superiorly, the whole hypotympanum is visualized and the trabeculae, cells, and finiculus are identified. The Arnold nerve coming out from the finiculus is noted and passes over the promotory. It is the area of Type 1 glomus tumor (tympano jugular). All wells of hypotympanum are noted with 0-degree and 45-degree 0.4-mm endoscopes (-Fig. 7). 


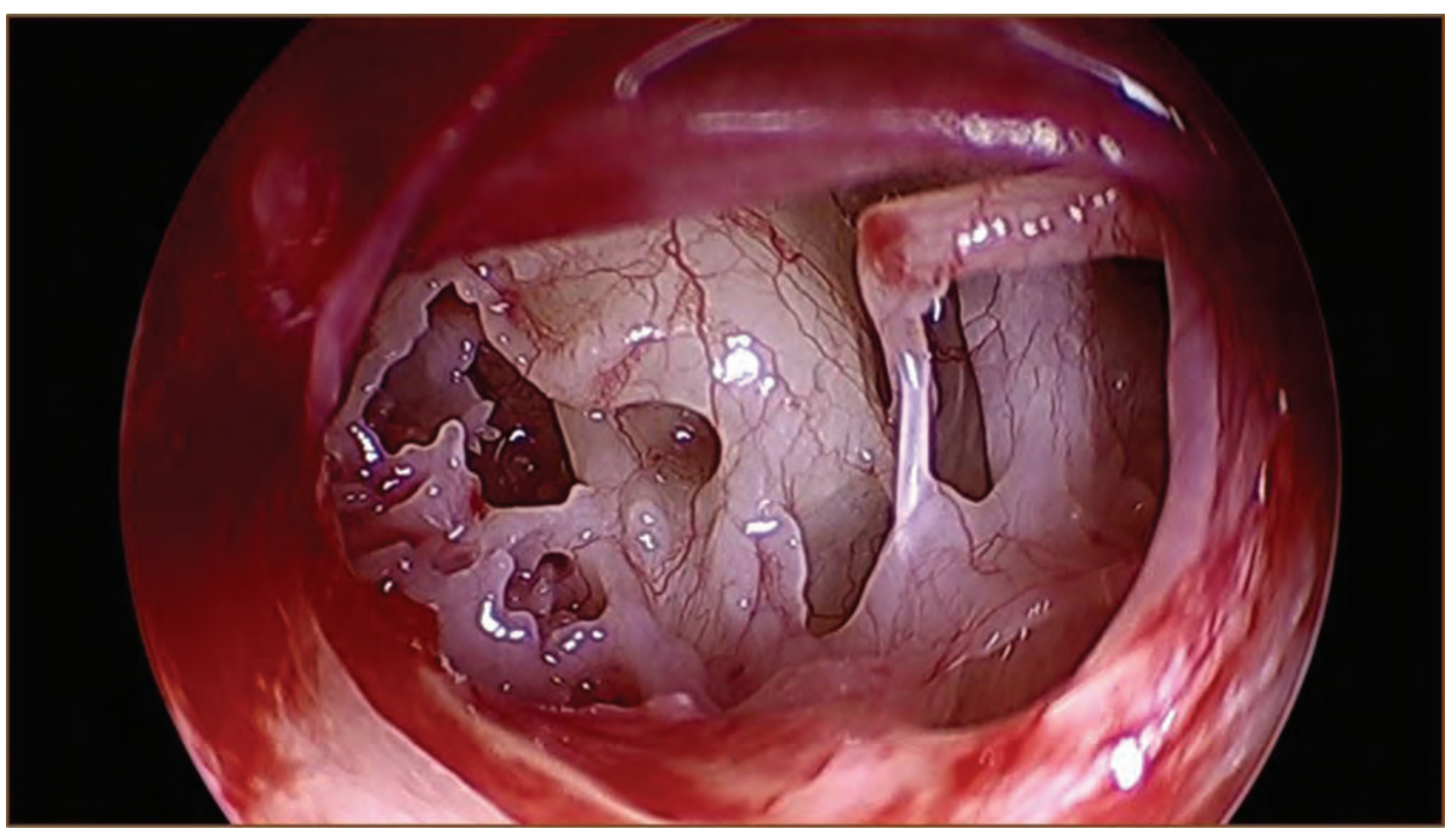

Fig. 4 Type A hypotympanum.



Fig. 5 Type B hypotympanum. 


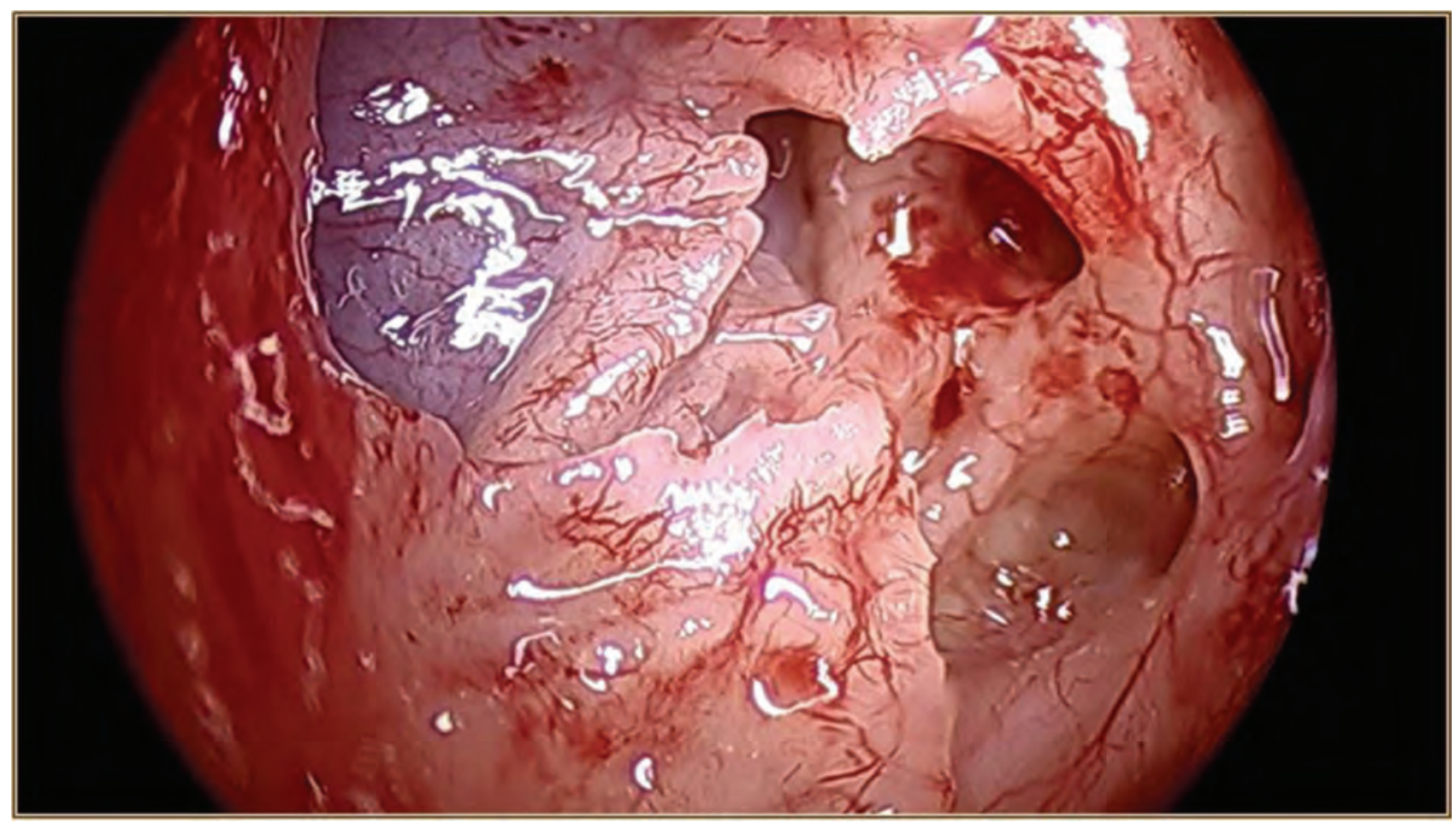

Fig. 6 Type C hypotympanum.

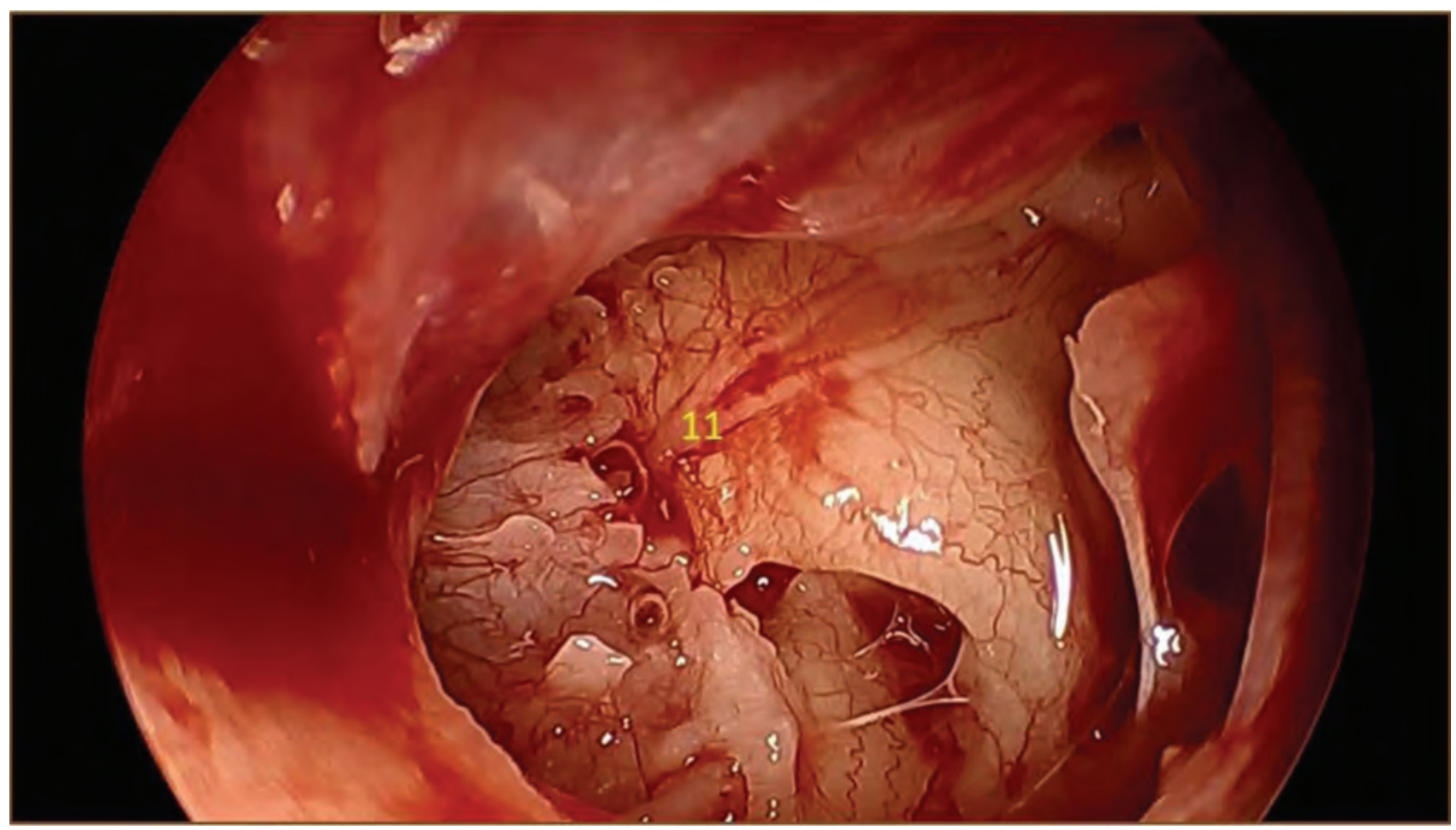

Fig. 7 Level 1. 11. Arnold's nerve. 


\section{Level 2}

After removal of all trabeculae, hypotympanotomy is performed leaving the inferior bony tympanic with using zero-degree endoscope transmeatally.

At this level, canalus tympanicus is exposed in the bone. The canalus tympanicus is a bony canal transmitting the Arnold nerve and the inferior tympanicus. It is the site for the origin of type 2 (glomus tympanicus), i.e., tympanojugular paraganglioma (-Fig. 8).

\section{Level 3}

Once the level 2 dissection is over using a 5-mm Diamond burr, the inferior bone along with the floor of the hypotympanum is drilled slowly. This exposes the Jugular bulb, Crista, the connective tissue band of Seibermann, and the crotch ( - Figs. 9-12).

\section{Level 4}

Once the crotch is carefully removed at the lower level, the jugular bulb posteriorly and internal carotid artery anteriorly are exposed. Pushing the jugular bulb posteriorly helps to visualize the glossopharyngeal nerve first, after separation of glossopharyngeal nerve from the surrounding connective tissue. The Vagus and Accessory nerves are exposed and the other structures of jugular foramen are visualized (-Figs. 13-15).

\section{Conclusion}

Hypotympanum is so far a neglected space in otological surgery. Recently, after the use of endoscopes transmeatal, the hypotympanum is clearly visualized with its minute details, including finiculus, trabeculae, Arnold nerve, hypotympanic cells and anterior and posterior hypotympanic sinuses. Endoscopic hypotympanum is divided into three types: type A, type B, and type C. In our observation, Type A hypotympanum is more common followed by type $C$ and type B. Our endoscopic endomeatal cadaveric dissection of hypotympanum exposes the jugular foramen with its contents transmeatally. It may be helpful for future endoscopic otological surgeries, that is limited jugular foramen tumors and type B glomus tympanicum tumors.

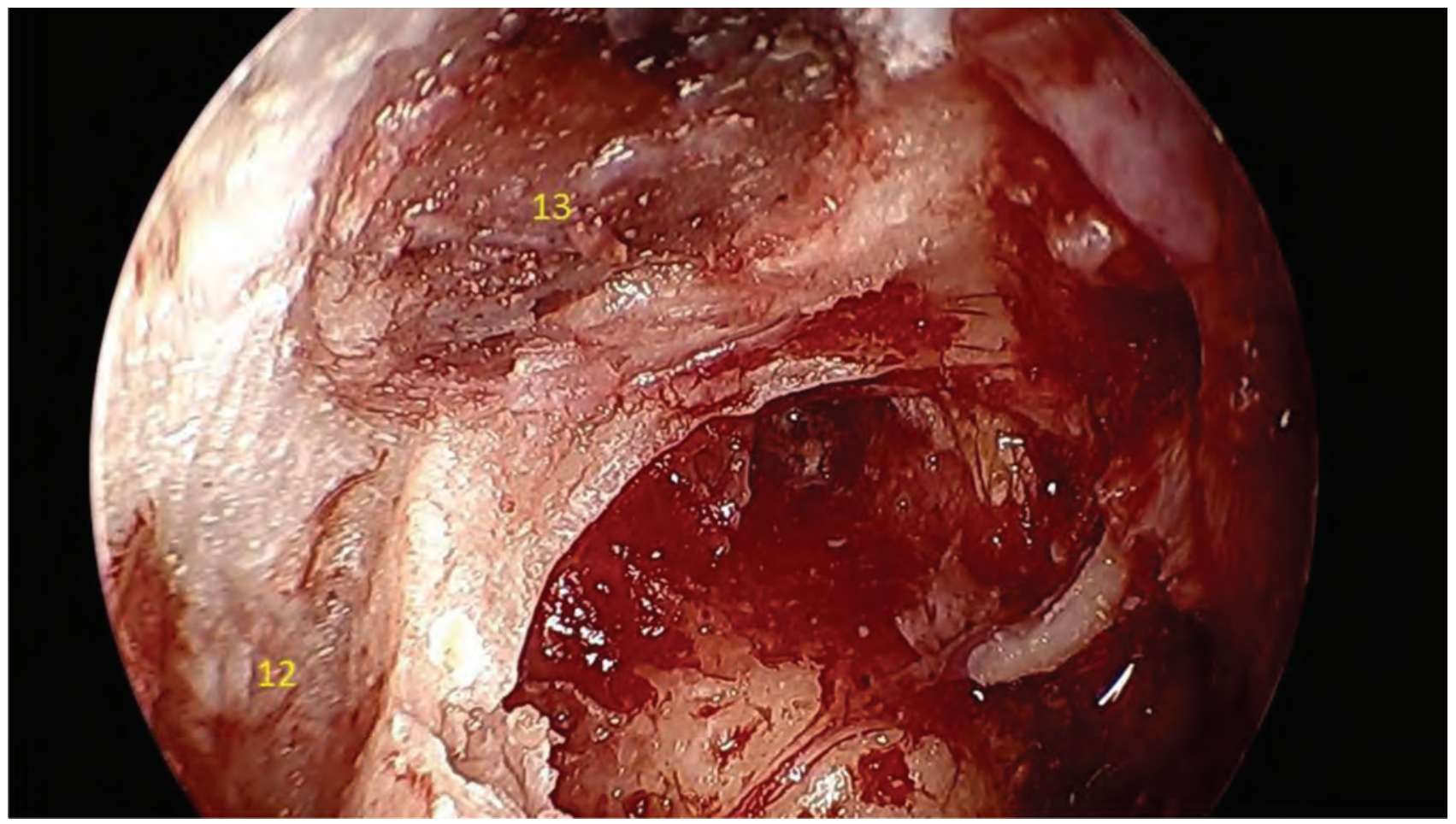

Fig. 8 Level 2. 12. Canalis tympanicus, 13. Temporomandibular joint. 




Fig. 9 Level 3. 14. Jugular bulb.



Fig. 10 Level 3 crista. 15. Crista. 




Fig. 11 Level 3 band of Seibermann. 23. Connective tissue band of Seibermann.

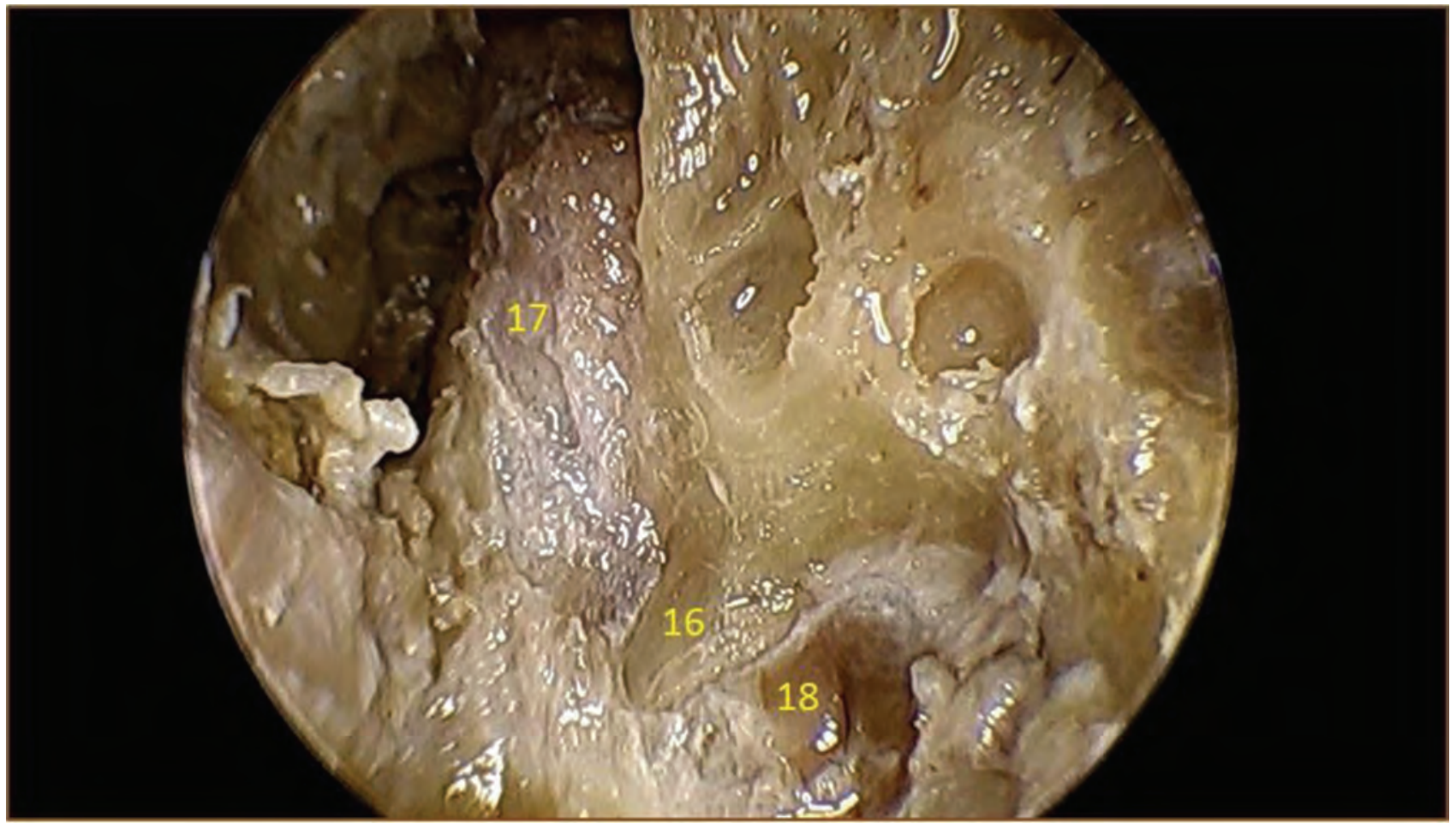

Fig. 12 Level 3 crotch. 16. Crotch, 17. ICA (internal carotid artery),18. Jugular bulb. 


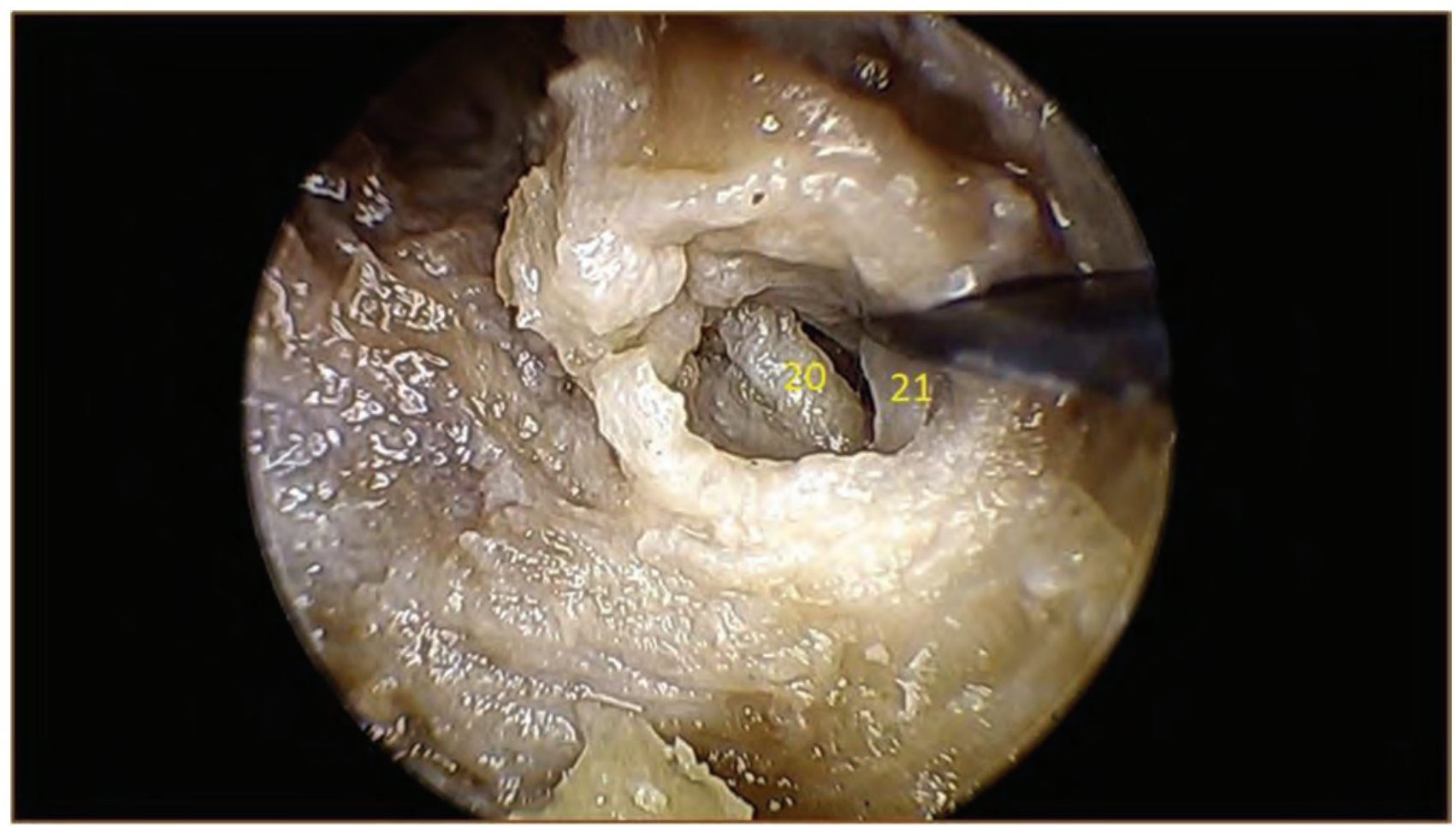

Fig. 13 Level 4 glossopharyngeal nerve. 20. Vagus nerve, 21. Accessory nerve.

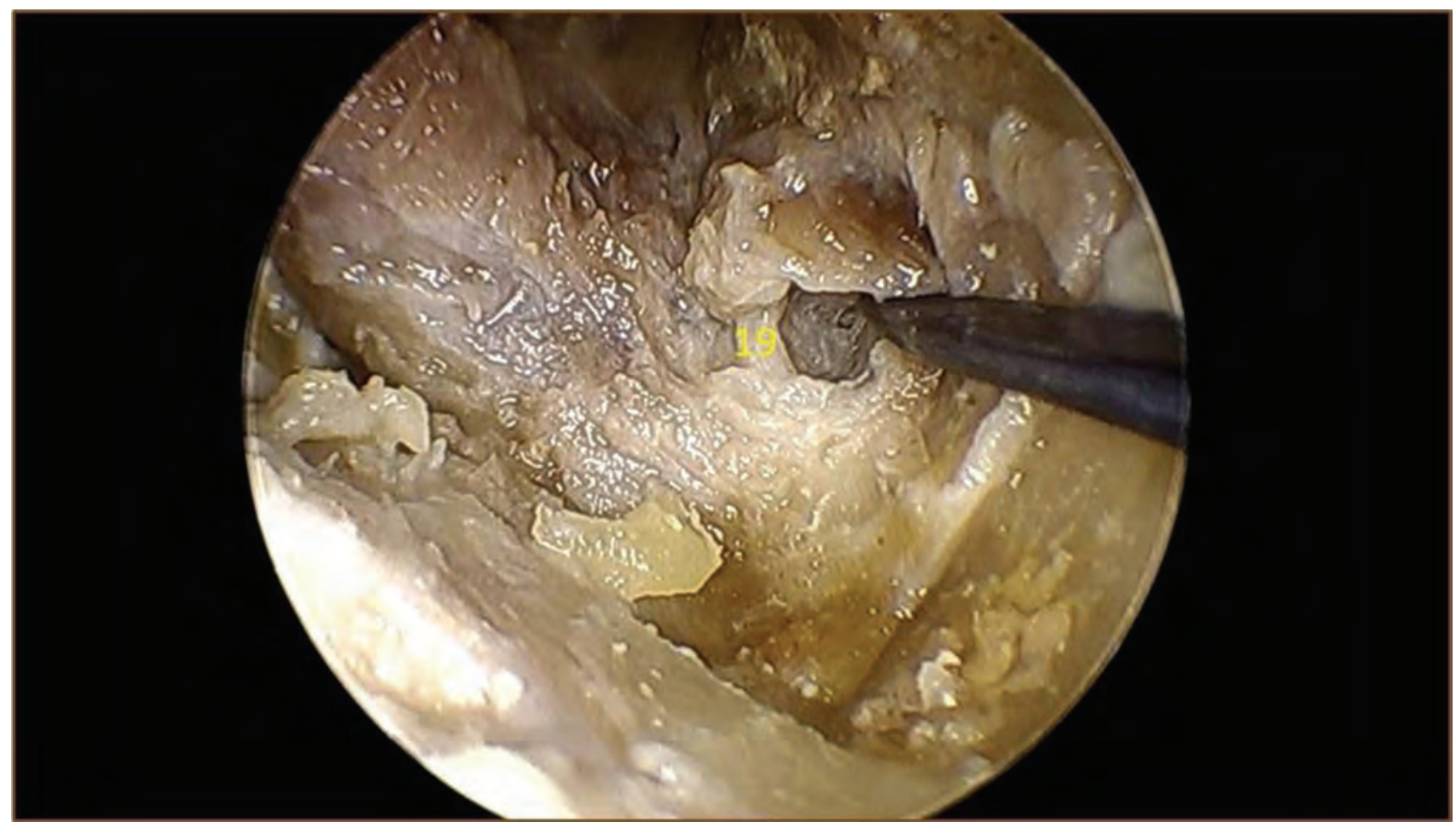

Fig. 14 Level 4 opening of jugular bulb. 19. Glossopharyngeal nerve. 


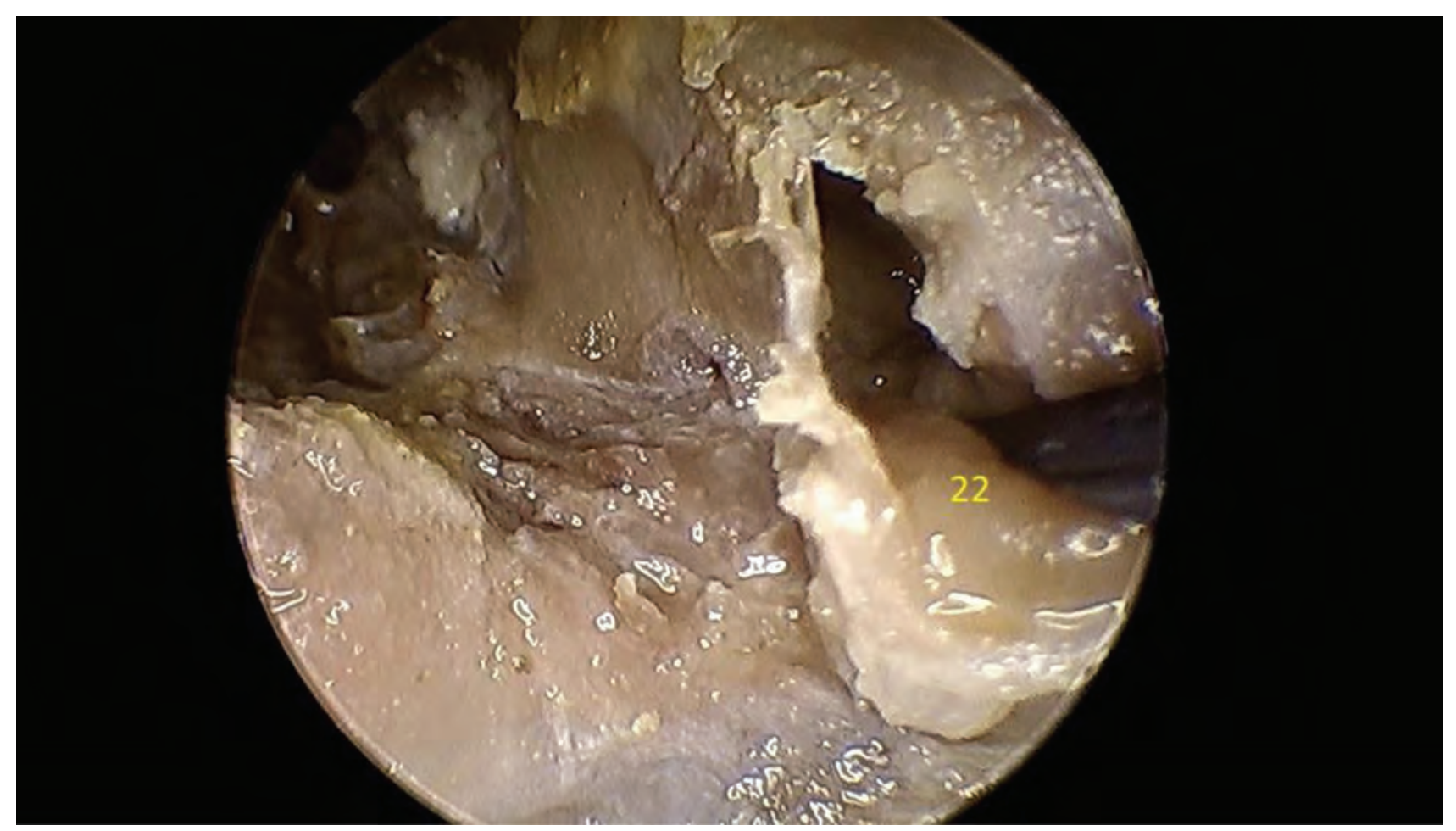

Fig. 15 Level 4 vagus. 22. Opening of Jugular bulb.

\section{Conflict of Interest}

None declared.

\section{References}

1 Savic D, Djeric D. Anatomical variations and relations of the medical and lateral portions of the attic and their surgical significance. J Laryngol Otol 1987;101(11):1109-1117

2 Turgut S, Tos M. Correlation between temporal bone pneumatization, location of lateral sinus and length of the mastoid process. The Journal of Laryngology and Otology 1992;106(6):485-489
3 Tarabichi M, Marchioni D, Presutti L, Nogueira JF, Pothier D. Endoscopic transcanal ear anatomy and dissection. Otolaryngol Clin North Am 2013;46(2):131-154

4 Turgut S, Tos M. Relation between temporal bone pneumatization and jugular bulb variations. In: Tos $M$, Thomsen J, eds. Acoustic neuroma. Amsterdam: Kugler, 1992 b: 257-261

5 Graham MD. The jugular bulb: its anatomic and clinical considerations in contemporary otology. Laryngoscope 1977;87(1):105-125 